\title{
Direct and indirect determination of electrocaloric effect in $\mathrm{Na}_{0.5} \mathrm{Bi}_{0.5} \mathrm{TiO}_{3}$
}

E. Birks, M. Dunce, J. Peräntie, J. Hagberg, and A. Sternberg

Citation: Journal of Applied Physics 121, 224102 (2017); doi: 10.1063/1.4985067

View online: http://dx.doi.org/10.1063/1.4985067

View Table of Contents: http://aip.scitation.org/toc/jap/121/22

Published by the American Institute of Physics

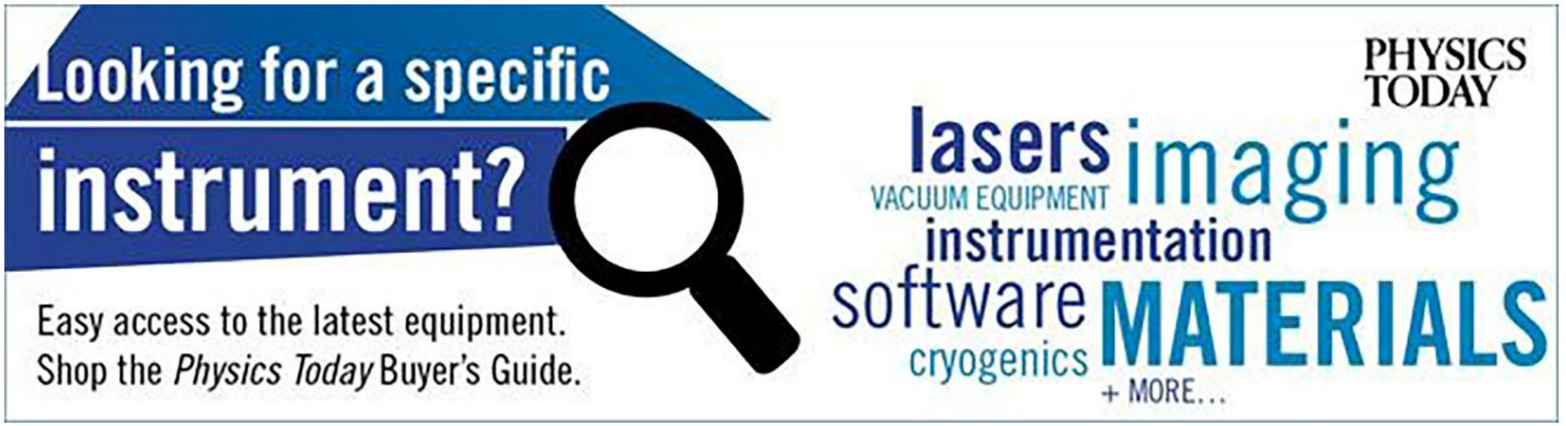




\title{
Direct and indirect determination of electrocaloric effect in $\mathrm{Na}_{0.5} \mathrm{Bi}_{0.5} \mathrm{TiO}_{3}$
}

\author{
E. Birks, ${ }^{1}$ M. Dunce, ${ }^{1, a)}$ J. Peräntie,${ }^{2}$ J. Hagberg, ${ }^{2}$ and A. Sternberg ${ }^{1}$ \\ ${ }^{1}$ Institute of Solid State Physics, University of Latvia, Kengaraga 8, Riga LV-1063, Latvia \\ ${ }^{2}$ Microelectronics and Materials Physics Laboratories, University of Oulu, P.O. Box 4500, FIN-90014 Oulu, \\ Finland
}

(Received 31 January 2017; accepted 24 May 2017; published online 8 June 2017)

\begin{abstract}
Direct and indirect studies of the electrocaloric effect were carried out in poled and depoled $\mathrm{Na}_{0.5} \mathrm{Bi}_{0.5} \mathrm{TiO}_{3}$. For this purpose, polarization and electrocaloric effect temperature change measurements were made at different electric field pulses as a function of temperature. The applicability of the widely used indirect electrocaloric effect determination method, using the Maxwell relation, was critically analyzed with respect to the reliable direct measurements. Quantitative differences were observed between the results obtained by both approaches in the case of the poled $\mathrm{Na}_{0.5} \mathrm{Bi}_{0.5} \mathrm{TiO}_{3}$ sample. These differences can be explained by the temperaturedependent concentration of domains oriented in the direction of the applied electric field. Whereas in depoled $\mathrm{Na}_{0.5} \mathrm{Bi}_{0.5} \mathrm{TiO}_{3}$, which is characterized by the electric field dependence of polar nanoregions embedded in a nonpolar matrix, the Maxwell relation is not applicable at all, as it is indicated by the obtained results. Possible mechanisms which could be responsible for the electrocaloric effect in the relaxor state were considered. The results of this study are used to evaluate the numerous results obtained and published by other authors, using the Maxwell relation to indirectly determine the electrocaloric effect. The reason for the negative values of the electrocaloric effect, obtained in such a way and widely discussed in the literature in the case of $\mathrm{Na}_{0.5} \mathrm{Bi}_{0.5} \mathrm{TiO}_{3}$, has been explained in this study. Published by AIP Publishing. [http://dx.doi.org/10.1063/1.4985067]
\end{abstract}

\section{INTRODUCTION}

After a long period of rather modest studies of the electrocaloric effect (ECE), which had left unfulfilled expectations of potential applications of ECE in cooling devices, this research direction has revived in recent years. It started from the publications of Mischenko ${ }^{1}$ and Neese, ${ }^{2}$ demonstrating a possibility to obtain much higher values of temperature changes, caused by ECE, $(\Delta \mathrm{T})$ compared to earlier results. Two characteristic features are inherent to the mentioned publications. First, the results were obtained for thin films and high electric fields, which are not accessible in bulk materials. Second, instead of direct measurements, one of the Maxwell relations in the integral form was used to calculate $\Delta \mathrm{T}$ from the electric field $(\mathrm{E})$ and the temperature dependence of polarization $(\mathrm{P})$ in a certain temperature range

$$
\Delta T=-\frac{T}{c_{E}} \int_{E_{1}}^{E_{2}}\left(\frac{\partial P}{\partial T}\right)_{E} d E
$$

where $\mathrm{T}$ is the absolute temperature and $\mathrm{c}_{\mathrm{E}}$ is the heat capacity at a fixed electric field. Starting from this milestone, a huge number of works, devoted to studies of ECE, have been published. They mostly follow the same path-measurements of $\mathrm{P}(\mathrm{T}, \mathrm{E})$ and calculations of $\Delta \mathrm{T}$ according to Eq. (1). The choice of such an approach is quite natural. Measurements of polarization are much easier to perform, compared to direct ECE measurements. Moreover, although direct measurements

\footnotetext{
a) Author to whom correspondence should be addressed: marija.dunce@ cfi.lu.lv.
}

of the temperature change in thin films are successfully accomplished in some cases, they are very tricky, in general due to extremely small heat capacity and very good thermal contact with the substrate. , $^{3,4}$

$\mathrm{Na}_{0.5} \mathrm{Bi}_{0.5} \mathrm{TiO}_{3}$ (NBT)-based compositions in the concentration region of the rhombohedral phase and the morphotropic phase boundary (MPB), which are widely studied due to their promising piezoelectric properties, have also been recently considered with respect to ECE. ${ }^{5-17}$ Except for Refs. 11 and 17, all studies based on the indirect measurements of ECE revealed temperature regions with remarkable so-called negative ECE - a decrease in temperature if the electric field is applied and an increase in temperature if the electric field is removed.

Taking into account the large number of publications and very promising values of ECE obtained using the Maxwell relation, the validity of the Maxwell relation in concrete cases is an important question. The results of studies, devoted to this issue, create an impression that the application of the indirect method in most cases is justified. ${ }^{18-23}$ However, a relaxor state (sometimes just non-ergodic) or contribution of lattice energy at ultra-high electric fields is assumed to be the reason for the disagreement in some cases. ${ }^{15,24-28}$ Some considerations on the influence of measurement conditions on values of $\mathrm{P}(\mathrm{E})$ have also been discussed. ${ }^{29}$ Considering NBT-based compositions, only partial correspondence between direct and indirect ECE measurements has been found in NBT- $\mathrm{KNbO}_{3}$ and NBT$\mathrm{K}_{0.5} \mathrm{Bi}_{0.5} \mathrm{TiO}_{3}$ solid solutions in the region of $\mathrm{MPB},{ }^{9,11}$ while even the opposite signs of the effect have been

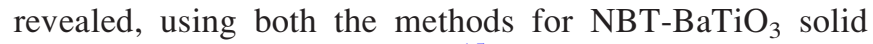
solutions in the region of MPB. ${ }^{15}$ 
The use of the Maxwell relations is very popular in studies of the magnetocaloric effect (MCE). The so-called giant MCE with values of magnetic field-induced entropy changes, even exceeding the theoretical limit available for a magnetic system, is obtained from the Maxwell relation in the case of the first order phase transition. ${ }^{30,31}$ Unfortunately, the reason for such large values sometimes is an incorrect application of the Maxwell relation. ${ }^{32}$ The conditions, under which using the Maxwell relations is not correct in studies of MCE, are also considered in Refs. 33 and 34.

This work is devoted to direct measurements of ECE in poled and depoled NBT and critical discussion of the applicability of the Maxwell relation [Eq. (1)] to evaluate ECE in ferroelectrics. Poled and depoled states in NBT are essentially different. While the poled NBT is in the ferroelectric state with symmetry R3c, ${ }^{35}$ the depoled state can be apparently characterized only by the coexistence of phases with different symmetry and corresponds to the behavior of relaxor ferroelectrics. ${ }^{36,37}$ Since the critical electric field $\left(\mathrm{E}_{\mathrm{c}}\right)$ between the relaxor and the ferroelectric states in NBT is high and a phase transition occurs in a narrow electric field range (inset on the left in Fig. 1), both states are well separated, which makes such a comparison reasonable.

\section{EXPERIMENTAL DETAILS}

$\mathrm{Na}_{0.5} \mathrm{Bi}_{0.5} \mathrm{TiO}_{3}$ (NBT) was synthesized with 2 mol. \% Bi over stoichiometry to compensate expected losses of $\mathrm{Bi}$ during processing. Besides depressing of conductivity at high temperatures, this also stimulates the reduction in $\mathrm{T}_{\mathrm{d} .}{ }^{38}$ NBT ceramics was prepared by the conventional solid state reaction method. Chemical-grade oxides and carbonates $\mathrm{Na}_{2} \mathrm{CO}_{3}, \mathrm{Bi}_{2} \mathrm{O}_{3}$, and $\mathrm{TiO}_{2}$ (purity $>99.5 \%$ ) were used as starting materials. The powders were weighed according to the formula, mixed with ethanol, and milled in an agate ball mill for $24 \mathrm{~h}$. The dried powders were calcined for the first time at $850^{\circ} \mathrm{C}$ for $2 \mathrm{~h}$. Afterwards, they were milled again in

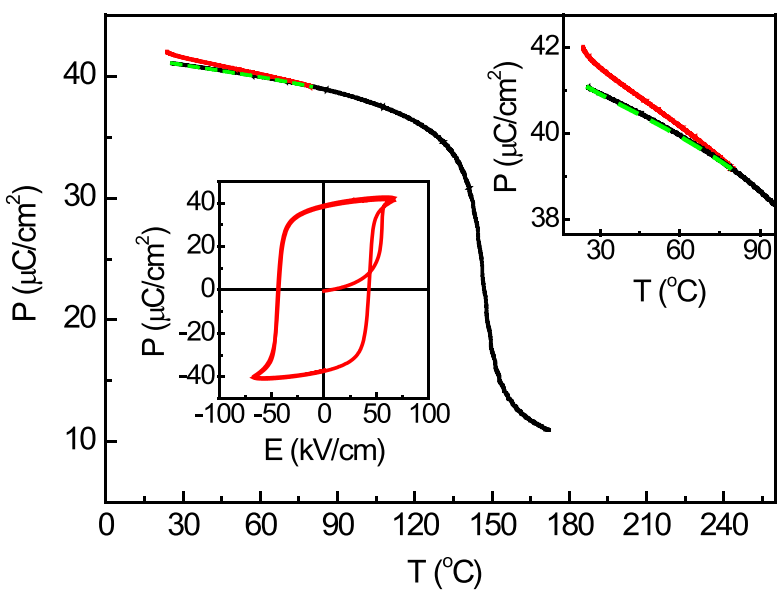

FIG. 1. Temperature dependence of the remnant polarization $\mathrm{P}_{\text {rem }}$, obtained from heating measurements of the sample poled at room temperature. Temporary alternation of the direction of the temperature change is also shown (a more detailed view in the inset on the right: first, the temperature was increased from room temperature to $70^{\circ} \mathrm{C}$-red solid line, then decreased to room temperature-green dashed line, and then increased till $170^{\circ} \mathrm{C}$-black solid line). Inset on the left: polarization hysteresis loop of a virgin NBT sample. the same conditions for $24 \mathrm{~h}$ and calcined for the second time at $1000^{\circ} \mathrm{C}$ for $2 \mathrm{~h}$. After milling in the same conditions for $24 \mathrm{~h}$ again, the powders were pressed uniaxially into a diskshaped pellet with a diameter of $\sim 17 \mathrm{~mm}$ and a height of $\sim 10 \mathrm{~mm}$ and then compacted under a pressure of $30 \mathrm{MPa}$ using a hydraulic press. The disk was sintered at $1200^{\circ} \mathrm{C}$ for $4 \mathrm{~h}$. In order to decrease evaporation of $\mathrm{Bi}$, calcination was carried out in covered platinum crucibles, whereas during the sintering, the disk was embedded in a powder of the same composition and placed under platinum crucibles on a platinum pallet.

A sample with a thickness of $0.3 \mathrm{~mm}$ was cut and polished. Electrodes were made by firing of Au paste at $500^{\circ} \mathrm{C}$ for ECE and polarization measurements. Polarization of the sample was performed by applying an electric field of $70 \mathrm{kV} /$ $\mathrm{cm}$ at room temperature. The same sample was used for measurements in the poled and depoled states. Measurements in the poled state were performed first. As a consequence, depolarization was ensured by heating till $280^{\circ} \mathrm{C}$.

During ECE measurements, the sample was connected with alumel wires and $3 \mathrm{M}$ polyimide adhesive tape on a sample holder. A chromel-alumel thermocouple was attached to one side of the sample on top of the polyimide adhesive for direct temperature measurements. The sample with the electrical wires was placed inside a closed beaker to ensure a long enough thermal time constant compared to the measurement period.

The response of the thermocouple, attached on the sample surface, was measured using a multimeter (34420A, Agilent Technologies, Inc.) while applying a $2.5 \mathrm{~s}$ long DC electric field pulse (including $0.125 \mathrm{~s}$ rise and fall times) from a function generator (33120A, Agilent Technologies, Inc.), connected with a high voltage amplifier (RT6000HVA, Radiant Technologies, Inc.). During the measurements, the polarization current through a shunt resistor was detected using an Agilent 34411A multimeter. The ECE temperature change was calculated from the thermocouple response by subtracting the thermocouple voltage response after the electric field pulse from the thermocouple voltage during the electric field pulse. Thus, the sign of $\Delta \mathrm{T}$ in the graphs presented further does not reflect the sign of $\Delta \mathrm{T}$ at a particular direction of the electric field change (increases on applying the electric field or decreases on removing the electric field). The sign is used to distinguish between the positive and negative ECE in terms characterized in the Introduction section.

The measurements were made by a step-like increase in temperature with a levelling time of $40 \mathrm{~min}$ at each temperature. Thermal loads of the electrodes and adhesive tape were estimated $(\sim 3 \%-7 \%)$ to correct the direct temperature measurements. All measurements were made under computer control in a Memmert UFP400 oven.

Additionally, polarization hysteresis $\mathrm{P}(\mathrm{E})$ measurements were performed using the Sawyer-Tower method in a quasistatic limit. The temperature dependence of the remnant polarization $\mathrm{P}_{\mathrm{rem}}(\mathrm{T})$ was measured after the measurement of $\mathrm{P}(\mathrm{E})$ on the same experimental setup, keeping $\mathrm{E}=0$, with a rate of temperature change of $3 \mathrm{~K} / \mathrm{min}$. 


\section{RESULTS AND DISCUSSION}

Temperature dependences of the directly measured ECE temperature change $\Delta T(T)$ for the poled and depoled NBT sample are presented in Fig. 2. In the case of the poled NBT sample, $\Delta \mathrm{T}(\mathrm{T})$ has a well-expressed maximum of $0.37^{\circ} \mathrm{C}$ (at electric field pulse $\mathrm{E}=20 \mathrm{kV} / \mathrm{cm}$ ) at a temperature of $\mathrm{T}_{\mathrm{m}}=150^{\circ} \mathrm{C}$, which corresponds to the temperature range, where depolarization takes place, and vanishes at temperatures above $210^{\circ} \mathrm{C}$ [Fig. 2(a)]. A slight decrease in $\mathrm{T}_{\mathrm{m}}$ can be observed upon lowering of the electric field. $\Delta \mathrm{T}(\mathrm{T})$ is positive in all temperature ranges where it is observed. Vanishing of $\Delta \mathrm{T}$ above $210^{\circ} \mathrm{C}$ apparently does not correspond to what could be expected in the paraelectric state, where a small, but detectable ECE temperature change should be observed in a wide range of temperatures above $T_{c}$ due to the temperature dependence of dielectric permittivity (the Curie-Weiss law). In the ferroelectric state, up to $130^{\circ} \mathrm{C}$, the ECE temperature change as a function of $\mathrm{P}_{\mathrm{i}}$, which is the electric field-dependent part of polarization at unipolar electric field pulses, at different temperatures follows a common path (Fig. 3) although $\mathrm{P}_{\mathrm{i}}(\mathrm{T})$ varies significantly upon changing the temperature. Such behavior was also observed earlier in NBT-containing solid solutions in the ferroelectric state. ${ }^{39}$

In the case of the depoled NBT sample, the maximum of $\Delta \mathrm{T}(\mathrm{T})$ is lower and more diffused than in the poled sample $-\Delta \mathrm{T}=0.14{ }^{\circ} \mathrm{C}$ at $\mathrm{T}_{\mathrm{m}}=142^{\circ} \mathrm{C}$ (at electric field pulse $\mathrm{E}=20 \mathrm{kV} / \mathrm{cm}$ ) [Fig. 2(b)]. Another difference is the change in the sign of $\Delta \mathrm{T}$ at temperatures above $210^{\circ} \mathrm{C}$, where $\Delta \mathrm{T}$ vanishes in the case of the poled NBT. The negative $\Delta \mathrm{T}$,

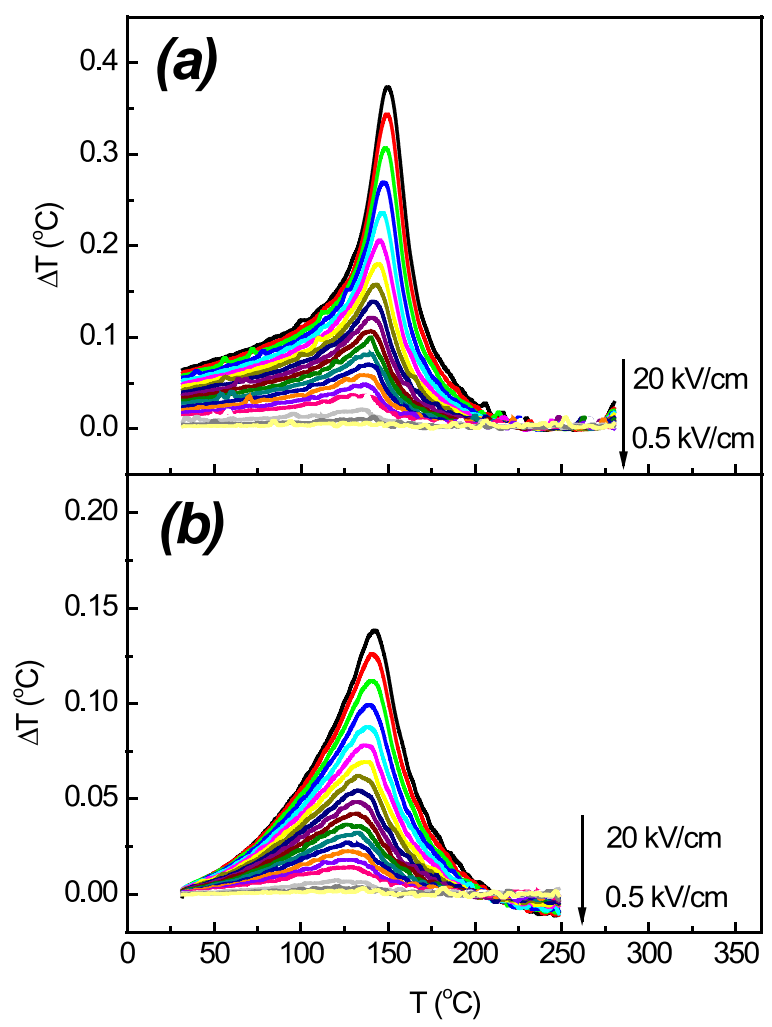

FIG. 2. ECE temperature change $\Delta \mathrm{T}(\mathrm{T})$ on removing the electric field at various electric field pulses for the poled (a) and depoled (b) NBT samples.

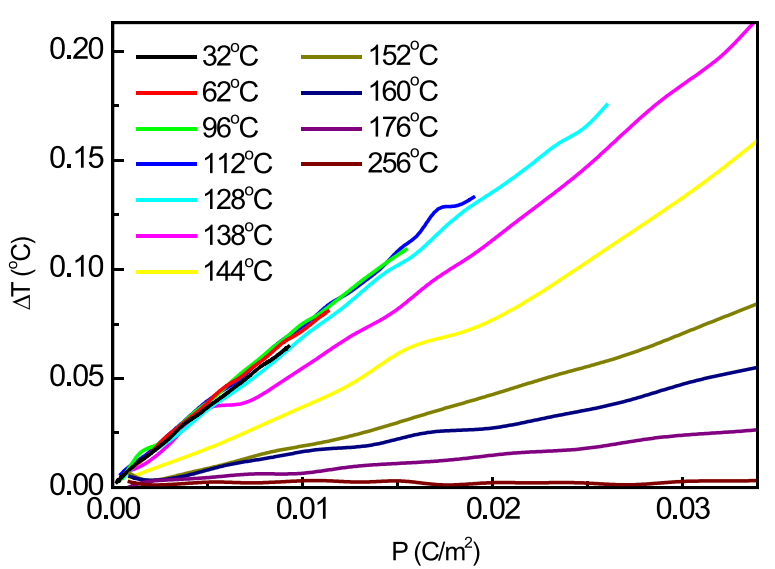

FIG. 3. ECE temperature change as a function of electric field-dependent part of polarization $\Delta \mathrm{T}\left(\mathrm{P}_{\mathrm{i}}\right)$.

observed upon removal of the electric field, is very small and, apparently, in $\Delta \mathrm{T}$ measurements upon application of the electric field, is masked by the Joule heat. Obviously, the different behavior indicates different states of the poled and depoled sample even in the temperature range, where the poled state is destroyed. A different behavior was also observed for the temperature and frequency dependences of dielectric permittivity. ${ }^{37}$ The dependence $\Delta \mathrm{T}\left(\mathrm{P}_{\mathrm{i}}\right)$ at different temperatures follows different paths and, in a wide temperature interval, can be considered as quasilinear with a slope which significantly decreases in the direction of lower temperatures (for the purpose of following analysis, it is illustrated as $\Delta \mathrm{T} / \mathrm{P}_{\mathrm{i}}$ versus electric field pulse value in Fig. 4).

The results of polarization measurements for the poled and depoled NBT samples, utilized to calculate ECE according to the widely used indirect method of ECE determination, are presented in Fig. 5. The temperature region was chosen far enough from the depolarization temperature $T_{d}$, where the nonlinearity of both $\mathrm{P}_{\mathrm{i}}(\mathrm{E})$ and $\Delta \mathrm{T}(\mathrm{E})$ is weak. Since the change in polarization was obtained simultaneously using the direct ECE measurements with a unipolar electric field change, in the poled state, it represents the change in $\mathrm{P}_{\mathrm{i}}(\mathrm{E})$ [Fig. 5(a)] instead of the total polarization $\mathrm{P}_{\text {tot }}=\mathrm{P}_{\text {rem }}+\mathrm{P}_{\mathrm{i}}$ (where $\mathrm{P}_{\text {rem }}$ is remnant polarization). In order

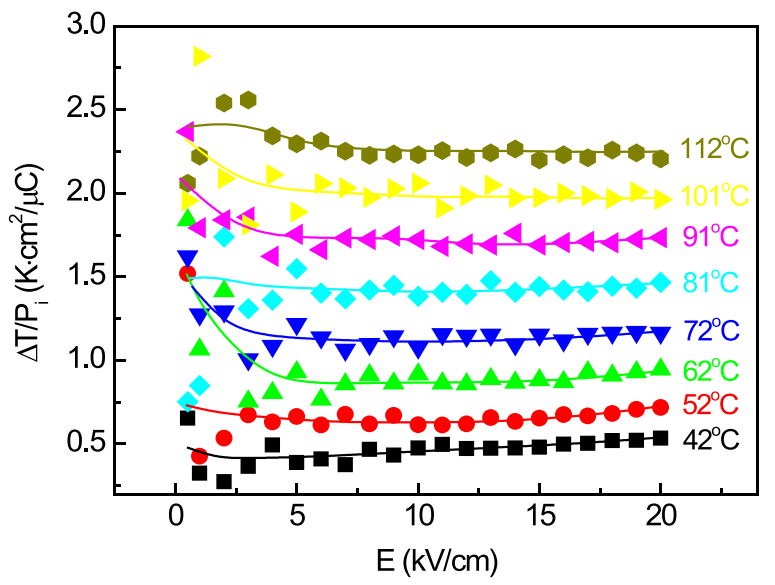

FIG. 4. ECE temperature change and polarization ratio $\Delta \mathrm{T} / \mathrm{P}_{\mathrm{i}}$ as a function of the electric field at various temperatures in the depoled state of NBT. 


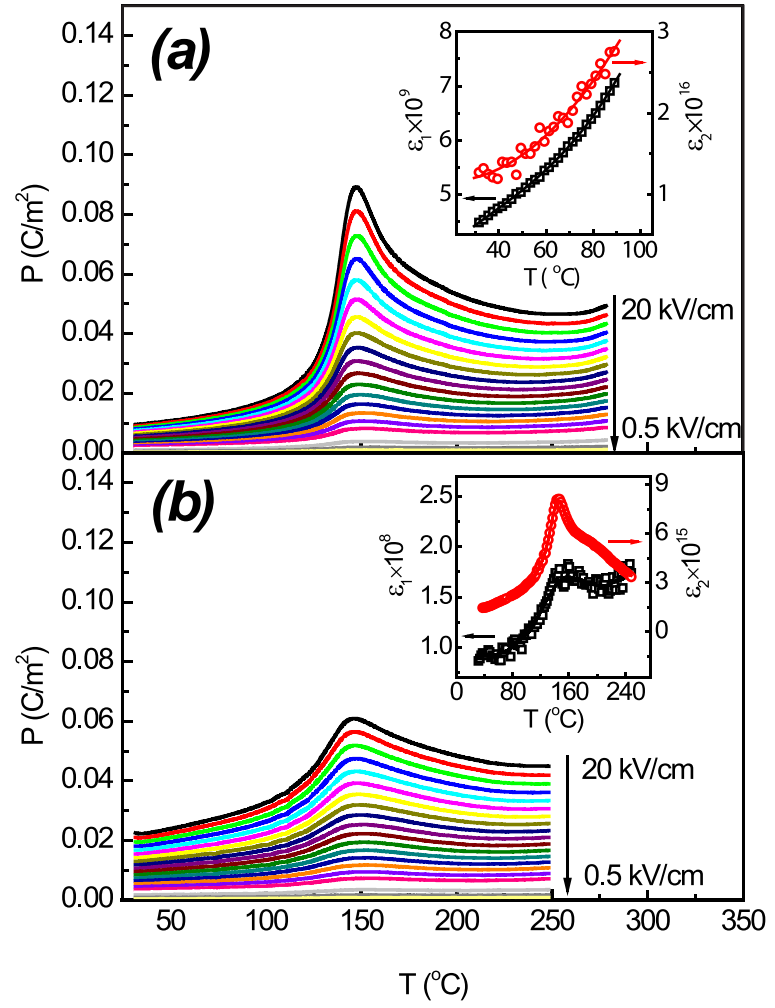

FIG. 5. Polarization as a function of temperature $P_{i}(T)$ at various unipolar electric field pulses for the poled (a) and depoled (b) NBT samples, measured simultaneously with ECE temperature change $\Delta \mathrm{T}$. The insets in (a) and (b) illustrate the temperature dependence of the extracted linear $\left(\varepsilon_{1}\right)$ and nonlinear $\left(\varepsilon_{2}\right)$ parts of dielectric permittivity for the poled and depoled NBT sample, respectively.

to obtain the total polarization, the results of $\mathrm{P}_{\text {rem }}(\mathrm{T})$ measurements were added (Fig. 1). $\mathrm{P}_{\text {rem }}$ smoothly decreases upon increasing the temperature, which is followed by a steep drop in the region of $\mathrm{T}_{\mathrm{d}}$, as it is expected in the poled state of NBT. For the calculation of the ECE temperature change, the measured $\mathrm{P}_{\text {rem }}(\mathrm{T})$ in the considered temperature range was replaced by the 3rd order polynomial fit, while $P_{i}(E)$ was replaced by the 2 nd order polynomial fit $\left(\mathrm{P}_{\mathrm{i}}(\mathrm{E})=\varepsilon_{1} \times \mathrm{E}+\varepsilon_{2}\right.$ $\times \mathrm{E}^{2}$ ), where the linear term represents the linear part and the 2 nd order term represents the nonlinear part of quasistatic dielectric permittivity. Both $\varepsilon_{1}$ and $\varepsilon_{2}$ are smoothly increasing functions of temperature [inset in Fig. 5(a)]. If dP/dT in Eq. (1) is replaced by the sum of all three contributions, the ECE temperature change can be calculated as follows:

$$
\Delta T=-\frac{T}{c_{E}}\left(\frac{d P_{r e m}}{d T} E+\frac{1}{2} \frac{d \varepsilon_{1}}{d T} E^{2}+\frac{1}{3} \frac{d \varepsilon_{2}}{d T} E^{3}\right) .
$$

Like in other published calculations of ECE in ceramics, the one-dimensional approach is used, assuming that spontaneous polarization $\mathrm{P}_{\mathrm{s}}$ and $\mathrm{E}$ are oriented in one direction and allowing one to simply replace $\mathrm{P}_{\mathrm{s}}$ by $\mathrm{P}_{\text {rem }}$. $\mathrm{P}_{\mathrm{i}}$ in such a case is purely induced (or intrinsic in terms used in Ref. 40) polarization. This issue will be discussed later. The values of temperature dependent $\mathrm{c}_{\mathrm{E}}$, necessary for the calculation of $\Delta \mathrm{T}$, were taken from Ref. 10.

Now, the contribution of the different terms to the ECE temperature change will be compared. Since the slope of
$\mathrm{dP}_{\text {rem }}(\mathrm{T})$ is negative, the contribution of this term to $\Delta \mathrm{T}$ is positive, while the contribution of both linear and nonlinear parts of dielectric permittivity is negative. The contribution of the nonlinear part of dielectric permittivity to $\Delta \mathrm{T}$ is negligible, while the contribution of the linear part is insignificant at low fields but reaches up to $12 \%$ of the total $\Delta \mathrm{T}$ at $\mathrm{E}=20 \mathrm{kV} / \mathrm{cm}$ in the considered temperature range. The calculated $\Delta \mathrm{T}$ values are larger than the directly measured values but can be aligned with them in the whole electric field and temperature range, where the comparison is made by simple rescaling and multiplying all calculated values roughly with 0.6 (Fig. 6). The role of $\mathrm{dP}_{\text {rem }} / \mathrm{dT}$ as a plausible reason for this difference can be illustrated in the following way. As it follows from Eq. (2), the slope of $\Delta \mathrm{T} / \mathrm{E}(\mathrm{E})$ at $\mathrm{E} \rightarrow$ 0 corresponds to $\mathrm{dP}_{\text {rem }} / \mathrm{dT}$. The directly measured $\Delta \mathrm{T}(\mathrm{E})$ was fitted by the polynomial function, and $\mathrm{dP}_{\text {rem }} / \mathrm{dT}$ was calculated from the linear term. The obtained results show approximately the same discrepancy between the calculated $\mathrm{dP}_{\mathrm{rem}} /$ $\mathrm{dT}$ and the one obtained from the measured temperature dependence of remnant polarization. Moreover, experimentally observed nonlinearity of $\Delta \mathrm{T}(\mathrm{E})$ is insignificant, which, according to Eq. (2), means that the whole value of $\Delta \mathrm{T}$ can be attributed to $\mathrm{dP}_{\mathrm{rem}} / \mathrm{dT}$, but $\mathrm{d} \varepsilon_{1} / \mathrm{dT}$ is insignificant.

The simplest way to explain the difference between the measured and calculated $\mathrm{dP}_{\text {rem}} / \mathrm{dT}$ is to assume that $\mathrm{P}_{\text {rem }}$ depends on the difference $\left(\mathrm{n}_{\mathrm{or}}\right)$ between concentrations of the domains oriented in the direction of the electric field and the domains oriented in the opposite direction (in the onedimensional approach), earlier considered in Ref. 25. So, the remnant polarization and pyroelectric coefficient, taking into account that $\mathrm{n}_{\text {or }}$ also can depend on temperature, can be expressed in the following way:

$$
\begin{gathered}
P_{r e m}=n_{o r}(T) \times P_{s}(T) \\
\frac{d P_{r e m}}{d T}=n_{o r}\left(\frac{\partial P_{s}}{\partial T}\right)_{n_{o r}}+P_{s}\left(\frac{\partial n_{o r}}{\partial T}\right)_{P_{s}} .
\end{gathered}
$$

In contrast to the earlier assumption, now, $P_{\text {rem }}$ and $P_{s}$ are mutually different since the presence of domains is taken

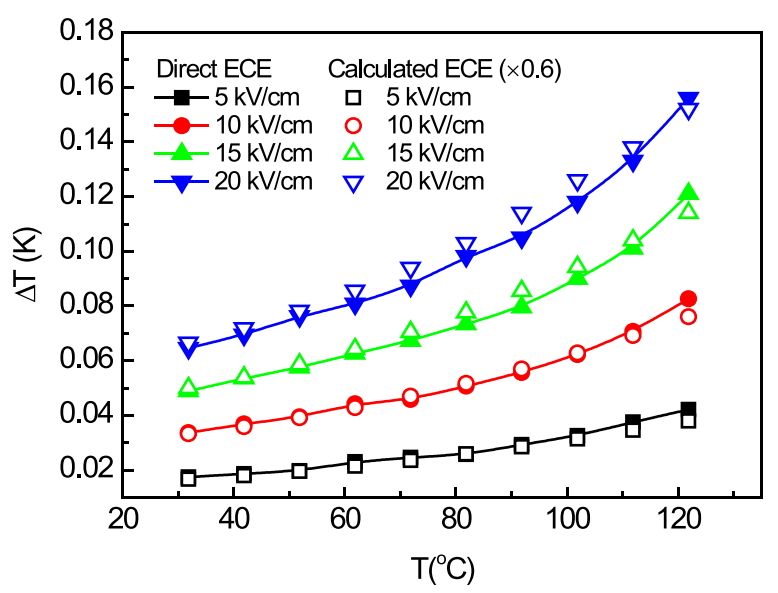

FIG. 6. Comparison between the directly measured ECE temperature change $\Delta \mathrm{T}(\mathrm{T})$ and $\Delta \mathrm{T}(\mathrm{T})$, calculated from Eq. (2) at four different values of electric field pulses $(5,10,15$, and $20 \mathrm{kV} / \mathrm{cm})$ for the poled NBT. The calculated values are multiplied with 0.6 . 
into account when calculating $\mathrm{P}_{\mathrm{rem}}$. While measurements of thermally stimulated depolarization and $\mathrm{P}(\mathrm{E})$ loops at $\mathrm{E}=0$ reflect $\mathrm{P}_{\text {rem }}$, only the first term in Eq. (4) contributes to ECE. Therefore, the difference between the directly and indirectly measured $\Delta \mathrm{T}$ is caused by the temperature dependence of $\mathrm{n}_{\mathrm{or}}(\mathrm{T})$. In order to illustrate the possible contribution of the second term in Eq. (4), let us assume that $\mathrm{P}_{\mathrm{s}}(\mathrm{T})=$ const in a certain temperature range, particularly between 25 and $125^{\circ} \mathrm{C}$. Simple calculations using Eq. (4) show that the experimentally observed decrease in $\mathrm{P}_{\text {rem }}(\mathrm{T})$ in the considered temperature interval corresponds to the decrease in $\mathrm{n}_{\text {or }}$ by $18 \%$.

The role of $n_{\text {or }}(T)$ can be confirmed by the irreversibility of $\mathrm{P}_{\text {rem }}(\mathrm{T})$ upon switching the direction of the temperature change (Fig. 1). It can be assumed that, during cooling, if it is started at a temperature $\mathrm{T} \ll \mathrm{T}_{\mathrm{d}}$, the domain configuration is more stable against the temperature change and $\mathrm{dP}_{\text {rem }}(\mathrm{T}) /$ $\mathrm{dT}$ approaches $\mathrm{n}_{\mathrm{or}} \times \mathrm{dP}_{\mathrm{s}} / \mathrm{dT}$. Indeed, $\mathrm{dP}_{\text {rem }} / \mathrm{dT}$ upon cooling is equal to $70 \%-80 \%$ from the value obtained upon heating and, to a large extent, reduces the discrepancy, previously observed between the calculated and directly measured ECE. It is remarkable that $\mathrm{P}_{\text {rem }}(\mathrm{T})$ upon subsequent heating follows the same path, which was observed upon cooling, before it joins the first heating curve (Fig. 1).

In spite of the difference between the results of the direct and indirect measurements of ECE, the fact that $\Delta \mathrm{T}\left(\mathrm{P}_{\mathrm{i}}\right)$ in the ferroelectric state follows the same path independent of temperature (Fig. 3, similar behavior was observed in NBT-based solid solutions ${ }^{38}$ ) creates an impression that $\mathrm{P}_{\mathrm{i}}$ can be considered as a variable, which solely characterizes $\Delta \mathrm{T}$ in the ferroelectric state. As it follows from the Ginzburg-Devonshire theory: ${ }^{41}$

$$
\Delta T=-\frac{2 \pi T}{c_{E} C_{w}}\left(P_{t o t}^{2}-P_{s}^{2}\right),
$$

which, taking into account that

$$
P_{\text {tot }}^{2}=\left(P_{s}+P_{i}\right)^{2}
$$

can be rewritten as

$$
\Delta T=-\frac{2 \pi T}{c_{E} C_{w}}\left(2 P_{s} P_{i}+P_{i}^{2}\right) .
$$

Here, $\mathrm{C}_{\mathrm{w}}$ is the Curie-Weiss constant. The part of Eq. (5.3) before the brackets is almost temperature-independent in the considered temperature range (far enough from $\mathrm{T}_{\mathrm{d}}$ ). Unfortunately, the value of the Curie-Weiss constant, which can be extracted from the nonlinear $\Delta \mathrm{T}\left(\mathrm{P}_{\mathrm{i}}\right)$ dependence as a tangent at $\mathrm{P}_{\mathrm{i}}=0\left(\mathrm{C}_{\mathrm{w}}=6.8 \times 10^{5} \mathrm{~K}\right)$, is remarkably larger than that for reference ferroelectric materials with the perovskite structure such as $\mathrm{BaTiO}_{3}{ }^{42}$ The reason for this inconsistence apparently is the contribution to $\mathrm{P}_{\mathrm{i}}$ of other components of the dielectric permittivity tensor in the three-dimensional system. Indeed, in contrast to $\mathrm{P}_{\text {rem }}$, which is rather close to $\mathrm{P}_{\mathrm{s}}$ after averaging over various directions of crystallographic axes of the rhombohedral lattice in ceramics $(87 \%$ of the value of $\mathrm{P}_{\mathrm{s}}^{43}$ ), the averaged value of $\mathrm{P}_{\mathrm{i}}$ contains the remarkable contribution of dielectric permittivity components in nonpolar directions. They usually are much higher than dielectric permittivity in the direction of $\mathrm{P}_{\mathrm{s}}^{44}$ and can even give the main contribution in the total value of $\mathrm{P}_{\mathrm{i}}$. The positive curvature of $\Delta \mathrm{T}\left(\mathrm{P}_{\mathrm{i}}\right)$, which contradicts with Eq. (5.3), can be also explained by the contribution of dielectric permittivity in nonpolar directions. For this reason, the estimation of $\mathrm{C}_{\mathrm{w}}$ from Eq. (4) is not possible even if the Ginzburg-Devonshire theory (in the three-dimensional case) is applicable.

When considering the measured $\mathrm{P}_{\mathrm{i}}(\mathrm{T})$ of the depoled sample [Fig. 5(b)], already at first glance, the Maxwell relation is not applicable in this case. $\mathrm{dP}_{\mathrm{i}} / \mathrm{dT}$ is positive below $\mathrm{T}_{\mathrm{m}}$, but $\mathrm{P}_{\text {rem }}=0$, unlike in the poled state. As a consequence, calculated $\Delta \mathrm{T}$ is purely negative at $\mathrm{T}<\mathrm{T}_{\mathrm{m}}$ (Fig. 7). Naturally, due to the maximum in the $\mathrm{P}_{\mathrm{i}}(\mathrm{T})$ dependence, the calculated $\Delta T(T)$ changes the sign in the region of $T_{m}$, becoming positive in a certain temperature region above $T_{m}$. Upon approaching $\mathrm{T}_{\mathrm{m}}, \varepsilon_{2}$ has a much more pronounced temperature dependence than $\varepsilon_{1}$ [inset in Fig. 5(b)], giving the main contribution to the calculated $\Delta \mathrm{T}$. The temperature region, where small negative directly measured $\Delta \mathrm{T}$ was observed (above $210^{\circ} \mathrm{C}$ ) [Fig. 2(b)], coincides with the temperature region, where $\mathrm{d} \varepsilon_{1} / \mathrm{dT}$ changes the sign and the correspondingly calculated contribution to $\Delta \mathrm{T}$ becomes negative again (inset in Fig. 7). For the total calculated $\Delta T(T)$, this temperature is shifted in the direction of high temperatures due to the contribution of $\mathrm{d} \varepsilon_{2} / \mathrm{dT}$ (Fig. 7). Since the nature of the electric field-induced polarization is rather complicated in the temperature range between $T_{d}$ and $T_{m}$, where relaxor properties continuously diminish and diffused phase transition to the orthorhombic phase is approached upon increasing the temperature, ${ }^{37}$ it is hard to comment such coincidence at the moment.

The nature of polarization in the depoled state of NBT is different from that of the poled state, as it should be considered as a relaxor state, ${ }^{37}$ consisting of polar nanoregions (PNRs) of rhombohedral structure, embedded in the nonpolar matrix. This means that, besides the induced polarization, $\mathrm{P}_{\mathrm{i}}(\mathrm{E})$ contains other relevant contributions, such as reorientation and breathing of PNR. ${ }^{45}$ Earlier, the breathing mechanism was used to interpret the frequency dispersion of

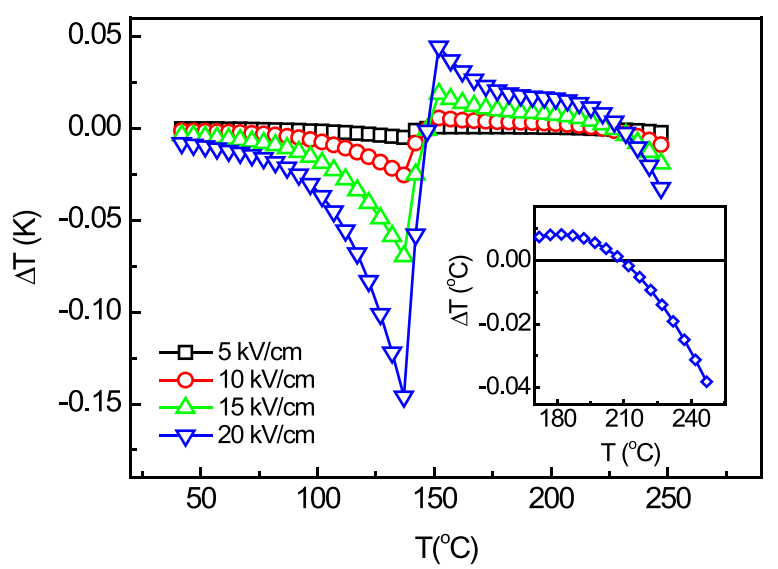

FIG. 7. Total ECE temperature change $\Delta \mathrm{T}(\mathrm{T})$ calculated according to Eq. (2) at four different values of electric field pulses $(5,10,15$, and $20 \mathrm{kV} /$ $\mathrm{cm}$ ) for the depoled NBT. The inset illustrates the solely contribution of $\mathrm{d} \varepsilon_{1} /$ dT to $\Delta \mathrm{T}(\mathrm{T})$ at a pulse of $20 \mathrm{kV} / \mathrm{cm}$. 
dielectric permittivity in NBT at low temperatures. ${ }^{46} \mathrm{~A}$ remarkable reduction in the $\Delta T\left(\mathrm{P}_{\mathrm{i}}\right)$ slope upon decreasing the temperature, expressed in Fig. 4 as $\Delta T / \mathrm{P}_{\mathrm{i}}$ as a function of $\mathrm{E}$, can be attributed to the PNR breathing mechanism, extended to moderate electric fields. Namely, the change in the PNR volume upon applying/removing the electric field essentially is a temporary local phase transition between the polar and nonpolar states. This corresponds to the increase in the volume $\left(\mathrm{v}_{+}\right)$for PNR oriented parallelly and the decrease in the volume $\left(\mathrm{v}_{-}\right)$for PNR oriented antiparallelly to the electric field. Let us assume that both $\mathrm{v}_{+}$and $\mathrm{v}_{-}$at a low enough electric field are linear functions of the electric field $-\mathrm{v}_{+}=\mathrm{m} \times \mathrm{E}$ and $\mathrm{v}_{-}=\mathrm{n} \times \mathrm{E}$. The contribution of the PNR volume change to $\Delta \mathrm{T}$ and polarization can be expressed in the following way:

$$
\begin{gathered}
\Delta T=-\frac{T}{c_{p}}\left[(m-n) E \Delta S^{P T}+\frac{1}{2} \frac{d P_{s}}{d T}(m+n) E^{2}\right] \text { and } \\
P_{i}=(m+n) E P_{s},
\end{gathered}
$$

where $\Delta S^{\mathrm{PT}}$ is the entropy jump at the electric field-induced phase transition. The contribution of the nonpolar matrix in Eq. (6), as well as intrinsic induced polarization of PNR and nonpolar phases in Eq. (7), is neglected. If the first term in Eq. (6) dominates (if $\mathrm{m} \gg \mathrm{n}$ ), $\Delta \mathrm{T}(\mathrm{E})$ is linear, while in case if only the second term contributes (if $\mathrm{m}=\mathrm{n}$ ), $\Delta \mathrm{T}(\mathrm{E})$ is proportional to $\mathrm{E}^{2}$. Indeed, the reduction in $\Delta \mathrm{T} / \mathrm{P}_{\mathrm{i}}$, observed in the direction of low temperatures, can be explained by the reduction in the contribution to $\Delta \mathrm{T}$ from the electric fieldinduced phase transition, if the difference $m-n$ is reduced

$$
\frac{\Delta T}{P_{i}}=-\frac{T(m-n) \Delta S^{P T}}{c_{p}(m+n) P_{s}} .
$$

This ratio is also electric field-independent in accordance with the experiment (Fig. 4).

The obtained results of direct ECE measurements in NBT are in deep contrast to the published results obtained using the indirect ECE determining method. ${ }^{5,7,8,14}$ The negative $\Delta \mathrm{T}$ of NBT, obtained by measurements of bipolar polarization hysteresis loops in the ferroelectric state and by applying the Maxwell relation, can be explained by the increased resistance of domain reorientation to the change in the electric field if the temperature is lowered. ${ }^{9,25,47}$ The increase in the coercive field $\mathrm{E}_{\text {coerc }}$, usually observed in the direction of low temperatures, also reflects this trend. Like in the case of $\mathrm{P}_{\mathrm{rem}}(\mathrm{T})$ considered above, the contribution of $\mathrm{n}_{\mathrm{or}}(\mathrm{T})$ [Eq. (3)] can be used in the interpretation of $\mathrm{P}(\mathrm{T})_{\mathrm{E}=\text { const }}$, obtained from polarization hysteresis loops. This contribution is remarkable in case if $E_{\max }$ does not exceed $E_{\text {coerc }}$ much ${ }^{7,8,14}$ or in the case of inclined polarization hysteresis loops with weakly expressed saturation. ${ }^{5}$ In both cases, a completely poled state is not achieved and $P_{\text {rem }}$ depends on $E_{\max }$.

The contradiction between the directly and indirectly measured values of ECE should not be a surprise since the Maxwell relations, strictly speaking, are applicable only for homogeneous systems where electric field-induced polarization has purely induced (intrinsic) nature. In none of the large number of publications, where ECE is calculated from the Maxwell relation, this requirement is fulfilled due to the presence of domains or even worse-coexistence of PNR and nonpolar matrix in the case of relaxor ferroelectrics. Finding an answer to the relevant question-in which cases and why the Maxwell relations give appropriate results-is outside the scope of the present study. According to considerations presented in this manuscript, Eq. (1) leads to correct results in the ferroelectric phase, if the first term in Eq. (4) prevails and $\mathrm{n}_{\mathrm{or}} \approx 1$. The application of the Maxwell relation in the relaxor state is more controversial due to the presence of PNR. Agreement with the direct measurements in the case of $0.9 \mathrm{PMN}-0.1 \mathrm{PT}$ films ${ }^{23}$ is observed at large electric fields, where the contribution of PNR could have already exhausted. Since Eq. (1) is also valid at the first order phase transition, ${ }^{48}$ interpretation of ECE as a result of local or macroscopic field induced first order phase transition perhaps could be used to explain why, in some cases, the Maxwell equation gives a reasonable agreement with the direct measurements of ECE in relaxor ferroelectrics even below the high electric field limit. ${ }^{22}$

\section{CONCLUSIONS}

Directly measured ECE in the poled and depoled NBT is not in agreement with the results of indirect ECE measurements, obtained from polarization as a function of the applied electric field at different temperatures and measured simultaneously with the electrocaloric response. The reason for the disagreement in the ferroelectric state is the temperaturedependent concentration of domains oriented in the poling direction. The almost temperature-independent path of $\Delta \mathrm{T}\left(\mathrm{P}_{\mathrm{i}}\right)$ can be interpreted in the framework of the GinzburgDevonshire theory, even taking into account that values of the thermodynamic parameters cannot be extracted due to the contribution of the nonpolar direction components of the dielectric permittivity tensor to $\mathrm{P}_{\mathrm{i}}$.

In the relaxor state of NBT, the direct application of the Maxwell relation leads to negative ECE temperature change values, which essentially contradicts with the results of the direct ECE measurements. One of the various $\mathrm{P}(\mathrm{E})$ mechanisms, existing in the relaxor state that can be used to explain the behavior of $\Delta \mathrm{T}\left(\mathrm{P}_{\mathrm{i}}\right)$, is the movement of PNR boundaries.

The directly measured ECE in the poled and depoled NBT reveals remarkable differences from the earlier published results of the indirect measurements, where negative ECE temperature change values were observed. Due to different possible contributions to polarization inherent to inhomogeneous systems, the nature of polarization measured in a particular experiment should be evaluated before the application of the Maxwell relation.

\section{ACKNOWLEDGMENTS}

This work has been supported by the National Research Program in the framework of the project "Multifunctional Materials and composites, photonics and nanotechnology $\left(\right.$ IMIS $\left.^{2}\right) . "$

\footnotetext{
${ }^{1}$ A. S. Mischenko, Q. Zhang, J. F. Scott, R. W. Whatmore, and N. D. Mathur, Science 311, 1270 (2006)

${ }^{2}$ B. Neese, B. Chu, S.-G. Lu, Y. Wang, E. Furman, and Q. M. Zhang, Science 321, 821 (2008).
} 
${ }^{3}$ S. G. Lu, B. Rožič, Q. M. Zhang, Z. Kutnjak, X. Y. Li, E. Furman, L. J. Gorny, M. Lin, B. Malic, M. Kosec, R. Blinc, and R. Pirc, Appl. Phys. Lett. 97, 162904 (2010).

${ }^{4}$ S. Crossley, T. Usui, B. Nair, S. Kar-Narayan, X. Moya, S. Hirose, A. Ando, and N. D. Mathur, Appl. Phys. Lett. 108, 032902 (2016)

${ }^{5}$ X. C. Zheng, G. P. Zheng, Z. Lin, and Z.-Y. Jiang, J. Electroceram. 28, 20 (2012).

${ }^{6}$ S. Uddin, G.-P. Zheng, Y. Iqbal, R. Ubic, and J. Yang, J. Appl. Phys. 114, 213519 (2013)

${ }^{7}$ W. P. Cao, W. L. Li, D. Xu, Y. F. Hou, W. Wang, and W. D. Fei, Ceram. Int. 40, 9273 (2014).

${ }^{8}$ X. Jiang, L. Luo, B. Wang, W. Li, and H. Chen, Ceram. Int. 40, 2627 (2014).

${ }^{9}$ F. Le Goupil, J. Bennett, A.-K. Axelsson, M. Valant, A. Berenov, A. J. Bell, T. P. Comyn, and N. M. Alford, Appl. Phys. Lett. 107, 172903 (2015).

${ }^{10}$ Y. Bai, G. P. Zheng, and S. Q. Shi, Mater. Res. Bull. 46, 1866 (2011).

${ }^{11}$ F. Le Goupil and N. M. Alford, APL Mater. 4, 064104 (2016).

${ }^{12}$ G. P. Zheng, S. Uddin, X. Zheng, and J. Yang, J. Alloys Compd. 663, 249 (2016).

${ }^{13}$ Q. Li, J. Wang, L. Ma, H. Fan, and Z. Li, Mater. Res. Bull. 74, 57 (2016).

${ }^{14}$ W. P. Cao, W. L. Li, X. F. Dai, T. D. Zhang, J. Sheng, Y. F. Hou, and W. D. Fei, J. Eur. Ceram. Soc. 36, 593 (2016).

${ }^{15}$ O. Turki, A. Slimani, L. Seveyrat, G. Sebald, V. Perrin, Z. Sassi, H. Khemakhem, and L. Lebrun, J. Appl. Phys. 120, 054102 (2016).

${ }^{16}$ M. Zannen, A. Lahmar, B. Asbani, H. Khemakhem, M. El Marssi, Z. Kutnjak, and M. Es Souni, Appl. Phys. Lett. 107, 032905 (2015).

${ }^{17}$ W. P. Cao, W. L. Li, T. R. G. L. Bai, Y. Yu, T. D. Zhang, Y. F. Hou, Y. Feng, and W. D. Fei, Ceram. Int. 42, 8438 (2016)

${ }^{18}$ P. D. Thacher, J. Appl. Phys. 39, 1996 (1968).

${ }^{19}$ R. B. Olsen, W. F. Butler, D. A. Payne, B. A. Tuttle, and P. C. Held, Phys. Rev. Lett. 45, 1436 (1980).

${ }^{20}$ G. Sebald, L. Seveyrat, D. Guyomar, L. Lebrun, B. Guiffard, and S. Pruvost, J. Appl. Phys. 100, 124112 (2006).

${ }^{21}$ S. Kar-Narayan and N. D. Mathur, J. Phys. D: Appl. Phys. 43, 032002 (2010).

${ }^{22}$ F. Le Goupil, A. Berenov, A.-K. Axelsson, M. Valant, and N. M. Alford, J. Appl. Phys. 111, 124109 (2012).

${ }^{23}$ M. Vrabelj, H. Uršič, Z. Kutnjak, B. Rožič, S. Drnovšek, A. Benčan, V. Bobnar, L. Fulanović, and B. Malič, J. Eur. Ceram. Soc. 36, 75 (2016).

${ }^{24}$ E. Birks, M. Dunce, and A. Sternberg, Ferroelectrics 400, 336 (2010).
${ }^{25}$ M. Dunce, E. Birks, J. Hagberg, J. Peräntie, M. Antonova, and A. Sternberg, Ferroelectrics 485, 143 (2015).

${ }^{26}$ S. G. Lu, B. Rožič, Q. M. Zhang, Z. Kutnjak, R. Pirc, M. R. Lin, X. Y. Li, and L. Gorny, Appl. Phys. Lett. 97, 202901 (2010).

${ }^{27}$ Y. Bai, G. P. Zheng, and S. Q. Shi, Appl. Phys. Lett. 96, 192902 (2010).

${ }^{28}$ M. Sanlialp, V. V. Shvartsman, M. Acosta, B. Dkhil, and D. C. Lupascu, Appl. Phys. Lett. 106, 062901 (2015).

${ }^{29}$ K. Ding, Y. Bai, X. Han, W. Zhang, and L. Qiao, Key Eng. Mater. 492, 164 (2012).

${ }^{30}$ A. Giguere, M. Foldeaki, B. Ravi Gopal, R. Chahine, T. K. Bose, A. Frydman, and J. A. Barclay, Phys. Rev. Lett. 83, 2262 (1999).

${ }^{31}$ J. S. Amaral and V. S. Amaral, Appl. Phys. Lett. 94, 042506 (2009).

${ }^{32}$ G. J. Liu, J. R. Sun, J. Shen, B. Gao, H. W. Zhang, F. X. Hu, and B. G. Shen, Appl. Phys. Lett. 90, 032507 (2007).

${ }^{33}$ J. S. Amaral and V. S. Amaral, J. Magn. Magn. Mater. 322, 1552 (2010).

${ }^{34}$ R. Niemann, O. Heczko, L. Schultz, and S. Fähler, Int. J. Refrig. 37, 281 (2014).

${ }^{35}$ B. N. Rao, R. Datta, S. S. Chandrashekaran, D. K. Mishra, V. Sathe, A. Senyshyn, and R. Ranjan, Phys. Rev. B 88, 224103 (2013).

${ }^{36}$ B. N. Rao, A. N. Fitch, and R. Ranjan, Phys. Rev. B 87, 060102 (2013).

${ }^{37}$ E. Birks, M. Dunce, R. Ignatans, A. Kuzmin, A. Plaude, M. Antonova, K. Kundzins, and A. Sternberg, J. Appl. Phys. 119, 074102 (2016).

${ }^{38}$ Y. S. Sung, J. M. Kim, J. H. Cho, T. K. Song, M. H. Kim, and T. G. Park, Appl. Phys. Lett. 98, 012902 (2011).

${ }^{39}$ M. Dunce, E. Birks, J. Peräntie, J. Hagberg, M. Antonova, R. Ignatans, and A. Sternberg, Ferroelectrics 498, 94 (2016).

${ }^{40}$ D. A. Hall, J. Mater. Sci. 36, 4575 (2001).

${ }^{41}$ G. G. Wiseman and J. K. Kuebler, Phys. Rev. 131, 2023 (1963).

${ }^{42}$ G. Rupprecht and R. O. Bell, Phys. Rev. 135, A748 (1964).

${ }^{43}$ B. Jaffe, W. R. Cook, Jr., and H. Jaffe, Piezoelectric ceramics (Academic Press, London, New York, 1971).

${ }^{44}$ M. Davis, M. Budimir, D. Damjanovic, and N. Setter, J. Appl. Phys. 101, 054112 (2007).

${ }^{45}$ A. K. Tagantsev and A. E. Glazunov, Phys. Rev. B 57, 18 (1998).

${ }^{46}$ J. Petzelt, S. Kamba, J. Fabry, D. Noujni, V. Porokhonskyy, A. Pashkin, I. Franke, K. Roleder, J. Suchanicz, and R. Klein, J. Phys.: Condens. Matter 16, 2719 (2004).

${ }^{47}$ M. Dunce, E. Birks, J. Peräntie, J. Hagberg, M. Antonova, and A. Sternberg, IEEE Trans. Ultrason. Ferroelectr. Freq. Control 61, 1364 (2014).

${ }^{48}$ J. R. Sun, F. X. Hu, and B. G. Shen, Phys. Rev. Lett. 85, 4191 (2000). 